\title{
VIII
}

\section{SKIN AFFECTIONS COMMONLY MISTAKEN FOR SYPHILIS}

\author{
By HENRY C. SEMON, M.D., M.R.C.P.
}

To attain any degree of completeness in so wide a field as the above title presents, it will be necessary to consider the three accepted stages of syphilis seriatim, and to discuss briefly such dermatological conditions as may occasionally give rise to confusion with the better-known condition. To save space, no attempt will be made here to describe the physical signs of syphilis, which, it is assumed, are familiar to every reader of this Journal.

\section{I.-PRIMARY ChANCRE}

In all varieties of the primary genital chancre, the incubation period will give a clue to the diagnosis, and the ultra-microscope will usually clinch it. From the diagnostic standpoint, it is our most powerful ally. We all recognise, however, that it is not infallible, and that the previous application of antiseptics may stultify the indisputable evidence it might have afforded.

We are, therefore, sometimes thrown back and compelled to rely on our knowledge of clinical signs, until such time as we can summon the Wassermann test reaction to our aid.

In the case of the Hunterian chancre, the following dermatoses require differentiation :-

(a) Epithelioma.-In its early stages this may closely resemble a chancre. There may be a suggestive history of coitus, the ulcer may be both painless and indurated, and there may be a chain of palpable, firm glands in the inguinal region.

The majority of such cases will require the verdict of microscopic sections.

(b) Tuberculous ulceration is less apt to be mistaken for a chancre. It is almost always a complication of tuberculosis elsewhere, is chronic in development, with thin overhanging and only slightly indurated edges, and is usually 


\section{BRITISH JOURNAL OF VENEREAL DISEASES}

exceedingly painful. Frequency of micturition, from associated genito-urinary infection, would probably call attention to the diagnosis.

(c) Erythema multiforme is rarely manifest on the penis or vulva alone. Usually the typical lesions on the backs of the hands and on the lips will accompany the genital sore, but if this is isolated a mistaken diagnosis of syphilis may be made. The flat bluish nodule, very soft in consistency, and painful to palpation, is the preamble to ulcer formation. It is exceedingly slow in its evolution and tendency to heal.

(d) Scabies is not likely to be mistaken for syphilis, but a scabietic lesion which becomes indurated, and does not rapidly clear up with treatment, should be examined for T. pallidum.

(e) Suppuration in the small sebaceous follicles of the glans is usually multiple and acute. Pus is rapidly formed and discharged, and the small crypts which form should make a decision easy.

(f) Eczematous conditions, inflamed seborrhœea and psoriasis may all afford occasional difficulty. The presence of the typical lesions elsewhere will usually give the clue to the true nature of the condition, and light may also be thrown upon it by a preceding history.

Chancrum molle and the balanitis of gonorrhcal complications hardly fall into the category of skin affections, and will not be here considered.

The primary syphilitic papule has to be differentiated in our scheme from two dermatoses only, viz., a chronic indolent " boil" due to follicle infection, and an isolated lesion of lichen planus.

The latter is by no means unknown, and when it occurs isolated in this situation, does not produce the characteristic itching.

Its other characteristics are, however, preserved, and the flat, shiny, polygonal papule, or group of papules, which never exhibit a tendency to necrosis, should instigate a search for lesions elsewhere, especially on the buccal mucous membrane, where they may be present as flat, china-white streaks.

The syphilitic erosion sometimes resembles herpes progenitalis. The reason for this is that the delicate roof of a vesicle in this situation is so rapidly disintegrated that a superficial erosion is sometimes the only manifestation. 


\section{BRITISH JOURNAL OF VENEREAL DISEASES}

The absence of induration is again our chief standby, while additional aids are afforded by the tendency to multiplicity and grouping, and the usually rapid appearance of herpes lesions (three to four days) after coitus.

It need scarcely be added that delayed healing, or a tendency to become indurated, should at once be investigated by the ultra-microscope. The great value of this test, and the invariable appeal to it as a final arbiter in all cases of doubt, make it incumbent on all practitioners never to apply an antiseptic to a genital lesion until the diagnosis has been established.

\section{II.-The Secondary ERuptions}

A few salient points in the clinical appearances of secondary syphilis may be briefly recalled.

They are polymorphic, i.e., they do not conform to one type.

The mucous membranes are always affected, even though this is not clinically evident.

The invariable adenitis is not always sufficiently appreciated.

The most important and usually the earliest glands to be involved are the sub-occipital, which drain the postnasal space.

(The syphilitic gland is not "shotty," as so often described in text-books. It is smooth, globular, always enlarged, and elastic in consistency. The rate of enlargement is slow, and not comparable to the effects of other infections, hence pain is not a feature.)

(a) The Roseola-Pityriasis Rosea of Gibert.-The dermatosis most commonly labelled syphilis is pityriasis rosea. This seasonal eruption is a harmless, exfoliative, non-recurrent and self-limited (six to eight weeks) condition. Its exfoliative tendency is its most salient characteristic, and that which should at once differentiate it from syphilis, which, although it may scale slightly at the margin of a papule, never exhibits a marked tendency in this respect. The macule of pityriasis rosea is always flat, and may exhibit soft infiltration in acute or irritated cases, but never in the same degree as the syphilitic papule.

The lesions of pityriasis rosea are all of one type, viz., macular, scaly,-with the thin, lace-like, crenated scales attached at the periphery of each lesion; of a rosy or 


\section{BRITISH JOURNAL OF VENEREAL DISEASES}

yellowish-red colour, but never of the coppery, luetic hue. The centre of the larger lesions is sometimes fawn-coloured, the periphery remaining pink. This difference in colour is not seen in luetic lesions.

Too much stress must not be laid on the absence of the " herald patch" in making the diagnosis, nor yet upon the subjective associations, such as itching, which is a variable feature, and may be entirely wanting. Slight cervical adenitis and prodromal sore throat are occasionally met with in pityriasis rosea, and fever may also precede the eruption. They are usually so slight as to escape notice, and no stress can be laid upon them from the diagnostic standpoint. Pityriasis rosea very rarely occurs on the face, scalp, or the extremities. Its localisation is typically that part of the trunk covered by a man's vest.

Drug rashes are not infrequently mistaken for roseolar syphilis, because they are apt to follow the administration of cubebs and copaiba in gonorrhœa. These rashes are all very acute and markedly hyperæmic. They fade easily on pressure, and do not exhibit polymorphism. They appear with great rapidity, and fade with equal suddenness. Sometimes they are scarlatiniform in type, and the conjunctivæ may become injected at the same time. There is never associated adenitis, and a diagnosis should be easy if the possibility is thought of.

Seborrhoic Dermatitis.-A confusion with this disease is unlikely if it is remembered that it is a disease of the follicles, and occurs, therefore, with a predilection for the hairy regions and the middle line of the body. The greasy appearance which is so characteristic of sebaceous abnormalities, the tendency to bran-like scaling, the presence of marked seborrhoea capitis, of a dry or oily character, will usually put the physician on his guard. The mistake is, perhaps, most apt to be made when a corona seborrhoica at the frontal margin of the scalp is present. This is usually sheet-like in aspect, irritable, and of a more yellowish tinge than the coppery discrete nodulo-papular syphilide known as the corona veneris.

(b) Papular Syphilide.-A common trap is the papulopustular eruption of rosacea. The forehead, nose, cheeks, and chin are the favourite sites of this reaction to a gastric reflex. The colour is enough to distinguish it, being of a bright red tint, and there is, moreover, a history of 


\section{BRITISH JOURNAL OF VENEREAL DISEASES}

dyspepsia and post-prandial flushing. (N.B.-The two conditions may be present at the same time.)

There are few papular dermatoses in which the aspect at all resembles the widespread papular eruption of syphilis, with its associated adenitis, condylomata, and mucous membrane lesions.

Lichen planus, in its more acute manifestations, is usually protected in this respect by the very severe pruritus which accompanies it. Its colour is rose-red to lilac, and the primary lesion is a small, shiny, raised, polygonal papule, with a pronounced tendency to appear in groups which coalesce to form patches, especially in regions of pressure or friction, such as under waist-bands or garters. The mucous membranes are sometimes attacked, but there is never any ulceration. When a pustular element is added to the papules there may be some difficulty.

Scabies is the most prominent example of a dermatosis in which both symptoms and signs may obscure an underlying roseolar or papular syphilide. There is no true scabietic eruption. Such papules and pustules as are present are the result of scratching, and their localisation is very characteristic.

It includes the forearms and hands, the axillary folds, the umbilical area, the lower part of the buttocks, the penis and the thighs. The typical "runs" are found between the fingers, and on the ulnar borders of the wrists, the breast in women, and the penis in men. That a rash itches does not, therefore, exclude syphilis, although it is a helpful diagnostic point when other and more important factors have been considered as a whole. Every case should be examined stripped, and in daylight if possible, if the error is to be avoided.

Some cases of seborrhoic dermatitis may have a superficial resemblance to a papular syphilide. Seborrhœa is protean in its manifestations, but these are strictly confined to the follicle. The rash, moreover, is not polymorphic in the same patient. The elements consist, as a rule, of grouped follicular papules, and are prone to occur in " mid-line" situations, such as the forehead, the nasolabial grooves, the chin, the sternal and interscapular regions. The colour is yellowish and not coppery in hue, the infiltration is extremely superficial when present, and the eruption as a whole is usually scaly, or with a tendency 


\section{BRITISH JOURNAL OF VENEREAL DISEASES}

in that direction. It is almost superfluous to add that the mucous membranes and glands are not affected in this disease.

Before we pass on to consider the eruptions which are occasionally mistaken for tertiary syphilis, a word must be said in the matter of infantile dermatoses.

There is really only one in which the question of lues sometimes gives rise to difficulty, viz., intertrigo, in which term are included all the septic manifestations about the buttocks and groins of young infants.

A neglected intertrigo does occasionally imitate the more widely disseminated eruption of congenital syphilis, but its colour is of a bright red or pink tint, and it does not wander far from the moist area of groin and perinæum where it originally started.

An eruption associated with it on the palms and soles is highly significant of a syphilitic etiology, and a search for it should be made at the very first examination. The rapid response to simple local applications, proper cleanliness, and treatment of the gastro-intestinal disturbance which so frequently accompanies it will set doubts at rest almost as soon as the definite answer given by the W.R. reaction.

Just as the ultra-microscopic demonstration of treponemata decides, or helps to decide, the nature of genital sores, so also we have become accustomed to rely on indications provided by the W.R. It must, however, be remembered that a positive result does not necessarily prove that a given rash is syphilitic, for psoriasis and certain other diseases may afford positive W.R. in a percentage of cases, and, again, the patient may have latent syphilis and a non-venereal dermatosis at the same time. An instance of this is under my care at the present time.

When first I saw the patient at the hospital in June, I924, I made a tentative diagnosis of pityriasis rubra pilaris (an exceedingly rare and intractable affection of the hair, sweat and sebaceous follicles of the entire body, associated with a pronounced exfoliative tendency), and ending fatally sometimes by the subsequent development of pulmonary tuberculosis, pneumonia, etc.

In this case there are groups of prominent and inflamed follicles covering certainly three-quarters of the trunk and extremities, with the usual scaling tendencies and loss of 
hair. About the end of June he developed bilateral subacute irido-cyclitis, which was considered of toxic origin by the ophthalmic surgeon. The Wasserman reaction, however, proved to be ++ . Repetition of the test produced the same result, and we were therefore compelled to treat the syphilitic factor in his case, although our belief in our clinical judgment remained unshaken. The patient himself strenuously denied the possibility of a genital infection, and a congenital one is put out of court by his age, thirty-five. The iritis had cleared up before I began to give injections for his positive blood reaction, so that the therapeutic test could only be observed on the eruption. This has decidedly improved after four doses of N.A.B., but it has not involuted at anything like the speed one would have expected in a syphilitic manifestation. Moreover, it is well known that chronic toxic eruptions of most kinds are nearly always bencfited by arsenic, for a time at least.* It is only the further progress of the eruption that will finally decide the issue.

A similar train of surmise is engendered by a consideration of a case which I showed to the Medical Society for the Study of Venereal Disease some two years ago, in which -a typical psoriatic, and responding to the appropriate remedies - the W.R. was positive and remained so, in spite of a complete treatment and the absence of any syphilitic taint in his own or his family history.

(c) Tertiary Syphilis.- The following skin eruptions present appearances which at times very closely resemble the later forms of cutaneous syphilis : various tubercular and mycelial infections, e.g., sporotrichosis, actinomycosis, etc.; acneform and sycotic lesions, due to pus organisms ; ecthyma and varicose ulcers ; lichen planus ; lupus erthyematosus; psoriasis ; rodent ulcer. The list might be greatly extended, but it is proposed to discuss here only the commoner forms of tuberculosis cutis, and such of the others above mentioned as are apt to occur in general practice in this country.

Tuberculosis.-It is an accepted thesis that the destructive effects of the tubercle bacillus are much slower than those due to the treponeme of syphilis. Hence the dura-

* There are still traces of the eruption present (Jan. I925), after a prolonged course of anti-luetic treatment-a fact which seemingly negatives a syphilitic etiology. 


\section{BRITISH JOURNAL OF VENEREAL DISEASES}

tion of the disease in a given case is of prime importance in the diagnosis.

In lupus vulgaris the disease begins early in life, advances very slowly, and the progressive ulceration, while attacking skin and cartilage, tends to spare the bone. Lupus nodules tend to recur in an apparently healed scar. The tuberculous ulcer usually has a ragged, ill-defined, shelving and irregular edge, and is often painful.

The circinate character of the syphilitic cutaneous gummatous ulcer hardly needs $\epsilon$ mphasis, but a tubercular ulceration may have a soméwhat similar outline, and the diagnosis in this class of case may be left in doubt until a therapeutic test with salvarsan has been tried. (The W.R. sometimes fails to clinch the diagnosis in this type of case.)

Bazin's disease is another form of tuberculide which begins as a nodule in the hypoderm and extends to the surface, where it tends to ulcerate and produce lesions resembling gummata. The situation over the gastrocnemii, usually in young and otherwise healthy women, is so very characteristic for this chronic disease that a confusion with syphilis should not be made.

The acneform tuberculide is neither so rapid nor so intense in its effects as its luetic homologue. It is an extremely indolent papulo-pustule, with a predilection for the trunk, where, with the lichenoid variety, it forms a group of very interesting minor tubercular manifestations, most frequently seen in children.

The many other types of tuberculide met with are rare, and do not fall into our present category.

Pus Infections.- The most likely difficulty to arise in this group is the ecthymatous ulcer on the legs. This was frequently mistaken for gumma during the War, when pediculi and puttees made it a common affection in the trenches. Ecthyma always begins as a superficial impetiginous infection, and slow necrosis under a scab is responsible for an ulcer of markedly indolent type, with bluish unhealthy edges, and often associated with considerable pain. The scar left is nearly always deeply pigmented, but not so thin and papery as that usually following syphilis, which by reason of its hypodermic origin is more destructive than the superficial process here described.

The ordinary coccogenic sycosis is limited, like sebor- 


\section{BRITISH JOURNAL OF VENEREAL DISEASES}

rhœic dermatitis, to the hair follicle, and does not, like syphilis, invade the intervening skin or give rise to gummatous nodules. Tinea sycosis, on the other hand, is much more acute, and may give rise to œdema in the subcutis underlying the purulent folliculitis, thus producing a superficial resemblance to the nodule of syphilis.

Actinomycosis, by reason of its chronicity, the presence of "woody" infiltration, and the numerous narrow sinuses discharging the grains of clumped fungus, should render the cases comparatively easy to diagnose.

Sporotrichosis has points of close resemblance both to tubercle and syphilis. It may be either ulcerative or hypertrophic and fungating in character, and the diagnosis can be settled only by recovery of the fungus from the discharge.

Lichen planus is occasionally isolated in its manifestations. A single non-ulcerative lesion on the glans penis, not unlike a superficial chancre redux, and composed of softly infiltrated, grouped papules in the coronal sulcus, was lately a striking example of the confusion that sometimes arises. The patient, a man of fifty-two, had a positive W.R., and did not deny the luetic infection. The lesion consisted of a delicate ring of discrete papules, and was not affected by antisyphilitic treatment. Under the mistaken impression that it was a case of tinea, X-rays had been administered, and the lesion at once disappeared. (It should be stated that X-rays have no effect on tinea of the glabrous skin, and only act on ringworm of the scalp by virtue of their epilating power.) The case was one of lichen planus, and still recurs from time to time, involuting at once to small doses of the X-rays.

Psoriasis in its fixed and infiltrated types may occasionally give rise to difficulty. No attempt at diagnosis should be made until the covering scales have been removed. This procedure will almost certainly reveal the cicatricial tendency in cases of syphilis; psoriasis never produces scars.

I have seen cases of superficial epithelioma and rodent ulcer in which the clinical appearances suggested a gummatous ulceration.

One cannot always make the diagnosis from the appearance of the edges of such an ulcer, which may occur, of course, in a syphilitic patient. The exceedingly slow growth, the resistance to anti-specific treatment, and the 


\section{BRITISH JOURNAL OF VENEREAL DISEASES}

highly characteristic appearance of a section should exclude the possibility of mistakes in this connection.

The varicose ulcer, that bugbear of most out-patient clinics, should always be tested for a positive W.R. Even in negative cases the signs are sometimes benefited by mercury and iodides by the mouth.

Usually the ulcer is in the lower third of the leg, and there is obvious varicosity of the saphenous vein and its tributaries. Sometimes, however, this is not apparent, and although the ulcer is not clean-cut, and does not exhibit a wash-leather slough, syphilis is a frequent association. 